\title{
Surface deformation studies of Tenerife Island, Spain from joint GPS-DInSAR observations
}

\author{
Sergey Samsonov*†, Kristy Tiampo ${ }^{\dagger}$, Pablo J. González ${ }^{\ddagger}$, Juan Prieto ${ }^{\S}$, Antonio G. Camacho ${ }^{\ddagger}$ and José Fernández ${ }^{\ddagger}$ \\ ${ }^{*}$ GNS Science, 1 Fairway Drive, Avalon, Lower Hutt, New Zealand, Email: s.samsonov@gns.cri.nz \\ ${ }^{\dagger}$ Department of Earth Sciences, University of Western Ontario, London, Ontario Canada \\ ¥Instituto de Astronomía y Geodesia (CSIC-UCM), Plaza de Ciencias 3, 28040, Madrid, Spain \\ $\S$ Dpt. Ingeniería Topográfica y Cartografía, ETSI Topografía, Geodesia y Cartografía, UPM. Madrid, Spain
}

\begin{abstract}
This work presents results for the three-dimensional displacement field at Tenerife Island calculated from campaign GPS and ascending and descending ENVISAT DInSAR interferograms. The goal of this work is to provide an example of the flexibility of the technique by fusing together new varieties of geodetic data, and to observe surface deformations and study precursors of potential activity in volcanic regions. Interferometric processing of ENVISAT data was performed with GAMMA software. All possible combinations were used to create interferograms and then stacking was used to increase signal-to-noise ratio. Decorrelated areas were widely observed, particularly for interferograms with large perpendicular baseline and large time span. Tropospheric signal was also observed which significantly complicated the interpretation. Subsidence signal was observed in the NW part of the island and around Mount Teide and agreed in some regions with campaign GPS data. It is expected that the technique will provide better results when more high quality DInSAR and GPS data is available.
\end{abstract}

\section{INTRODUCTION}

Tenerife is the largest of the seven Canary Islands in the Canary Archipelago with the surface area of $2034 \mathrm{~km}^{2}$. The island is clearly divided to a very dry southern part and a very humid and vegetated north. Like the rest of the Canary Islands, it is of volcanic origin. The last stage of volcanic activity started about $500 \mathrm{~K}$ ago and the last eruption occurred in 1909. The eruptive system of the island is dominated by the Las Canadas Caldera and Teide, a 3715-m stratovolcano located at the northern border of the caldera which was formed during last 150,000 years (Figs. 1-2). For the past 100 years, volcanoes of the central volcanic complex (CVC) on Tenerife Island were quiescent, but a recent increase in seismic activity around the island [8] as well as diffuse emission of carbon dioxide along a zone known as the Santiago Rift [11] and increasing fumarolic activity at the summit of Teide volcano [7] suggest their reawakening.

In this work we applied a GPS-DInSAR optimization technique [13] which combines GPS and DInSAR data into a timedependent three-dimensional densely spaced deformation field in order to identify local and regional geodetic signals. For the first time, campaign GPS was used instead of continuous GPS data such as in our previous works of southern California [14], [15]. In this case the accuracy of the campaign GPS observations is significantly lower, but the importance of each observation is significantly higher due to the small number of

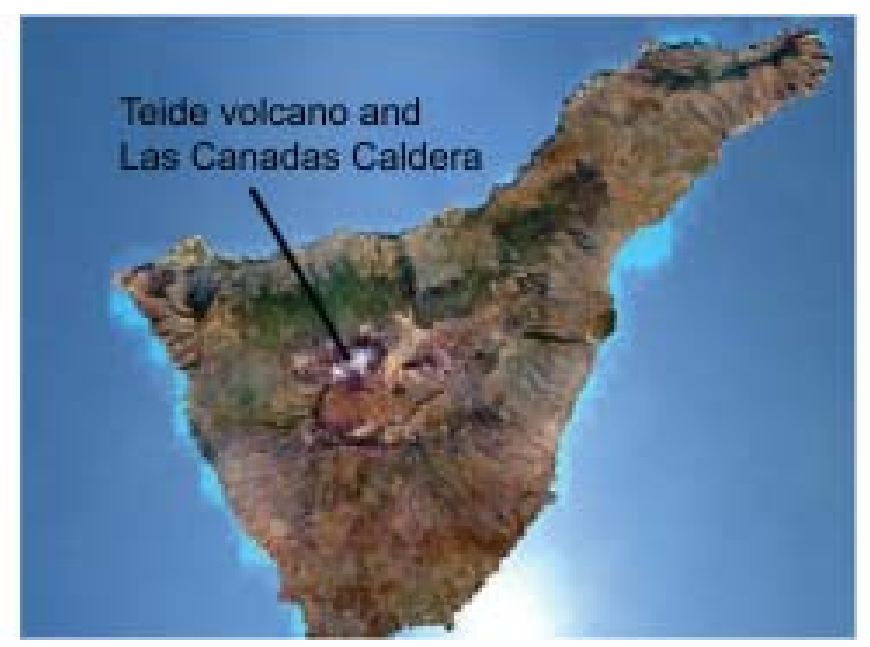

Fig. 1. The satellite image of the Tenerife Island. The Las Canadas Caldera as well as Teide volcano are well recognizable features on the island. Reprinted from ESA web site.

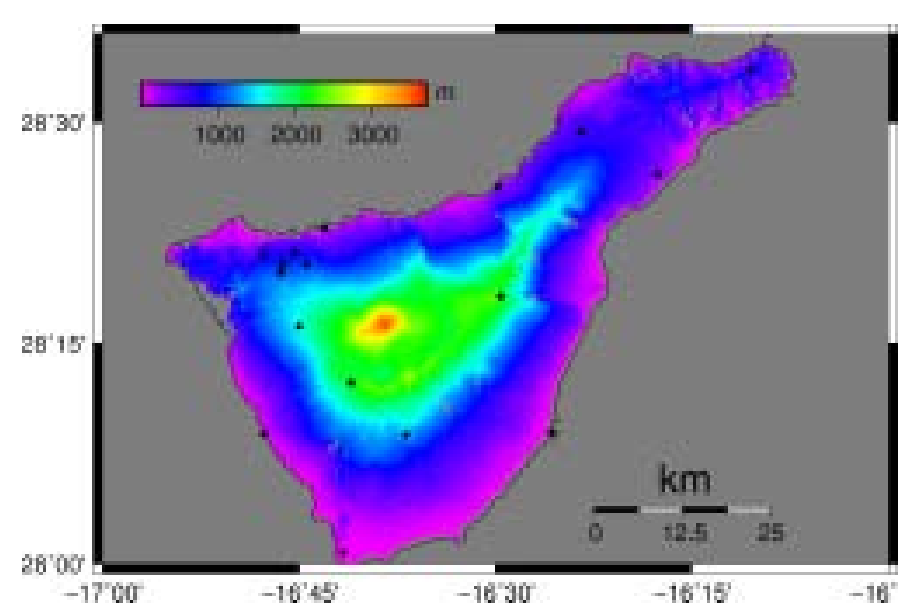

Fig. 2. The digital elevation model of the Tenerife Island which is used in this study. Black dots are the locations of GPS sites. 
observations and their targeted location. The lower accuracy of GPS observation affects the accuracy of the initial model and, therefore, the accuracy of final GPS-DInSAR model. In order to compensate for lower accuracy of the GPS data, two DInSAR images from ascending and descending orbits were used simultaneously in the optimization.

\section{DATA USED IN PREVIOUS AND CURRENT STUDIES}

For the past few decades a number of different studies were undertaken in this area in order to find precursors of possible volcanic eruptions. In particular, geodetic measurements in the southern part of the caldera using geodetic and leveling micronetworks [16] and seismic studies [10], several gravity campaign for structural studies [17], [1], [2], temporary observations of gravimetric tides [3], regular observations of temperature, fumarolic activity and measurements of different gases emitted at the top of Teide volcano [12] and diffusive degassing in and around the caldera [9]. These observations have not detected any clear anomaly that could be regarded as an indicator of volcanic reactivation in the monitored areas. However, since both historic and recent volcanic activities are very scattered [6] over the island it was suggested that new techniques covering the whole island need to be used, otherwise some anomalies associated with future eruptions might not be detected on time [18].

\section{A. Joint deformation and gravity observations}

Recent GPS and gravity measurement campaigns were undertaken to measure surface deformation and gravity changes as precursors of possible volcanic eruptions. The first joint deformation-microgravity survey was performed in May 2004, two weeks after an observed increase in seismic activity, followed by campaigns in July 2004, April 2005 and July 2005. The network consisted of 14 benchmarks operated with the accuracy of $0.01 \mathrm{mGal}$ and positioned in order to cover an area of approximately $500 \mathrm{~km}^{2}$ of the central volcanic complex, including the Pico Viejo - Pico Teide complex, the Las Canadas Caldera as well as the Santiago Rift. Within the accuracy of the measurements, surface uplift was observed only at four benchmarks [8]. A widespread deformation pattern was not observed by these surveys.

\section{B. Campaign GPS observations}

The first independent campaign GPS observations were performed in 2000 and were consequently repeated every year until 2006, skipping the year of 2003. The GPS observations were performed using geodetic double frequency receivers installed for a period of 2-5 hours (depending on the baseline length) in 2000, and for more than 6 hours in the later campaigns, independent of the baseline length. The collected data was processed using Bernese software [4] with precise ephemerides. Precision obtained during the survey was of the order of a few $\mathrm{mm}$ for horizontal components, and slightly less than $1 \mathrm{~cm}$ for the vertical component. Results for the interpolation of these measurements by ordinary kriging are presented in Figs. 4(a), 4(b) and 4(c) with corresponding errors 4(d), 4(e) and 4(f). The values of deformation here were converted to velocities by applying linear regression to the 2000-2006 time series in order to make them comparable to the differential InSAR velocity fields used in this technique. However, if required, these velocity fields can be easily converted to deformation fields by multiplication of the timespan.

\section{DInSAR observations}

Differential interferograms were calculated from Synthetic Aperture Radar (SAR) images acquired by the ERS-1/2 satellites starting from 1992. These images cover the whole island and provide continuous deformation pattern with a high spatial resolution of approximately $30 \times 30 \mathrm{~m}$ and an accuracy better than $1 \mathrm{~cm}$. Unfortunately some areas decorrelate due to surface changes and different look angles resulting in large perpendicular baseline and, therefore, can not be used for measurement of surface deformation.

These studies confirmed that there was no deformation in and around the Las Canadas Caldera, coinciding with similar results obtained from different terrestrial measurements. However, a few areas of deformation were identified in the northwest corner of the island which coincided with the region of the last historic eruption [5].

Beginning in 2003, a few scenes became available from ascending and descending passes of ENVISAT satellite (presented in Table I). However, only a few interferometric pairs with baseline less than 550 meters were successfully unwrapped for the whole island and used in this study (Fig 3).

\section{THE GPS-DINSAR OPTIMIZATION}

The GPS-DInSAR optimization technique was used to combine 2000-2006 campaign GPS and ENVISAT DInSAR data available for Tenerife Island. In general this methodology was similar to that used in our previous works [13], [15], [14], but with some modifications. First of all, GPS data was acquired only at certain times, but not continuously. In total there were six GPS campaigns with time intervals of approximately one year (with one exception in 2003). These observations were post-processed in order to account for a precise position of the satellites during acquisitions and to remove some common noise by using differential techniques. The tectonic plate velocity, common for all stations, was also subtracted from both horizontal components. The time series were created for each component of each GPS site and then this data was used to calculated velocities of deformations by applying linear regression.

Ordinary kriging interpolation method was used to calculate initial continuous velocity maps and corresponding errors with the same geocoding and resolution $(3 \mathrm{sec})$ as differential InSAR interferograms. Gamma ISP and DIFF/GEO software packages were used to calculate differential interferograms from SLC data. SRTM digital elevation model was used to remove the topographic phase and precise baselines were calculated by estimating local fringe rate using two-dimensional FFT transform and refined using least-squares estimations 


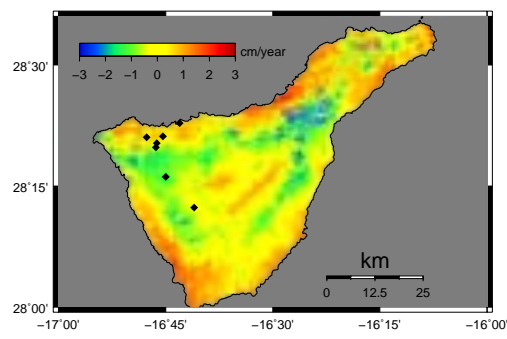

(a) 2003-2004 (asc)

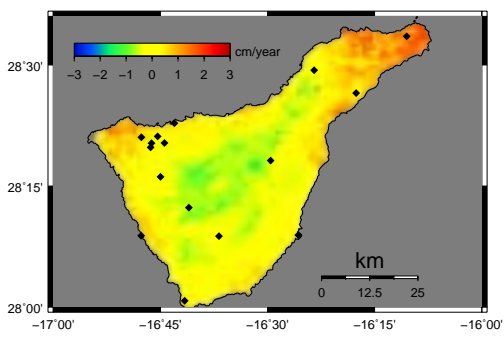

(b) 2003-2006 (asc)

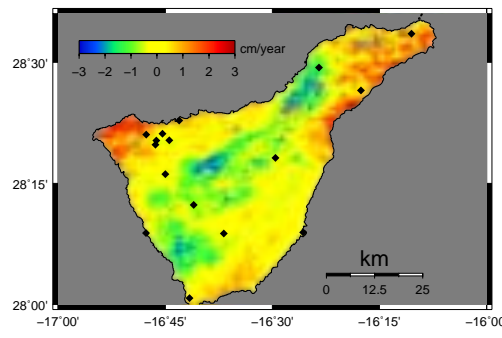

(c) $2005-2006$ (asc)

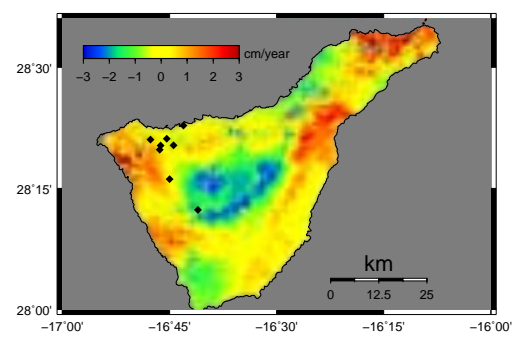

(d) 2004-2005 (asc)

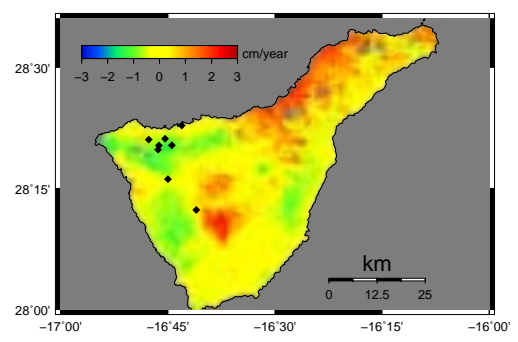

(e) 2004-2005 (dsc)

Fig. 3. Ascending and descending stacks of ENVISAT interferograms of Tenerive Island. The time span is shown above, black diamonds are campaign GPS sites for corresponding time periods.

TABLE I

Differential interferograms used in this study. Ascending interferograms are grouped in three partial stacks spanning approximately 2003-2004, 2004-2005, 2005-2006. Also two complete stacks were calculated: ascending 2003-2006 and descending 2004-2005.

\begin{tabular}{c|c|c|c|c|c}
\hline Ascending & Time span in days & $B_{p}, \mathrm{~m}$ & Descending & Time span in days & $B_{p}, \mathrm{~m}$ \\
\hline $20030907-20040613$ & 281 & 25 & $20040611-20050527$ & 350 & -377 \\
$20030907-20050320$ & 562 & 147 & $20040611-20050701$ & 386 & 384 \\
& & & $20040611-20050909$ & 452 & -536 \\
$20040613-20050320$ & 281 & 122 & $20050527-20050909$ & 102 & 324 \\
$20040613-20050703$ & 383 & -275 & $20050527-20051014$ & 138 & 67 \\
$20050213-20050807$ & 175 & 259 & $20050527-20051118$ & 171 & 351 \\
$20050320-20050703$ & 102 & 163 & $20050527-20051223$ & 208 & 144 \\
$20050424-20050703$ & 69 & 309 & $20050701-20050909$ & 65 & -437 \\
& & & $20050805-20050909$ & 32 & 514 \\
$20050213-20060129$ & 350 & -87 & $20050805-20051223$ & 138 & 334 \\
$20050213-20060305$ & 386 & 104 & $20050909-20051014$ & 36 & -254 \\
$20050529-20060129$ & 244 & -347 & $20050909-20051118$ & 69 & 27 \\
$20050807-20060129$ & 175 & -156 & $20050909-20051223$ & 105 & -180 \\
$20050807-20060305$ & 211 & 210 & $20051014-20051118$ & 32 & 281 \\
$20060129-20060305$ & 36 & 191 & $20051014-20051223$ & 69 & 74 \\
& & & $20051118-20051223$ & 36 & -206 \\
\hline
\end{tabular}

TABLE II

Four optimization runs performed for different timespans that were analyzed in this work. Five interferometric stacks were calculated: A1 (2 interferograms) 2003-2004, A2 (5) 2004-2005, A3 (6) 2005-2006, A4 (14) 2003-2006, D (16) 2004-2005.

\begin{tabular}{c|c|c|c|c|c}
\hline Run & Time period & Asc. pair & Dsc. pair & GPS time span & Num. of GPS stations \\
\hline 1 & $2003-2004$ & A1 & & $2002.65-2004.48$ & 7 \\
2 & $2004-2005$ & A2 & D & $2004.48-2005.57$ & 8 \\
3 & $2005-2006$ & A3 & & $2005.57-2006.13$ & 16 \\
4 & $2003-2006$ & A4 & D & $2002.65-2006.13$ & 16 \\
\hline
\end{tabular}

based on the ground control point data. All possible masterslave configuration of SAR data with perpendicular baseline less than 700 meters were processed but only 13 ascending and 16 descending interferograms with perpendicular baseline less than 550 meters were selected and used in this study (Table I).

Four ascending 3(a), 3(b), 3(c), 3(d) and one descending 3(e) interferometric stacks were calculated from processed 


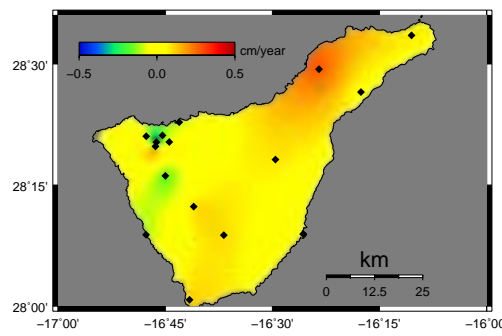

(a) north

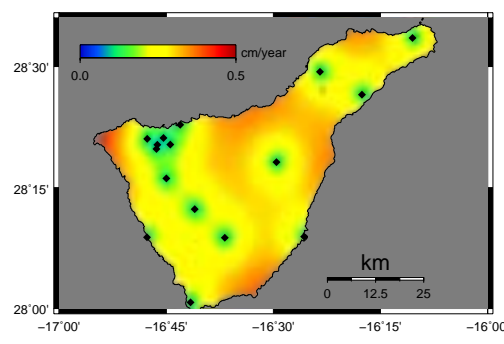

(d) north st. dev.

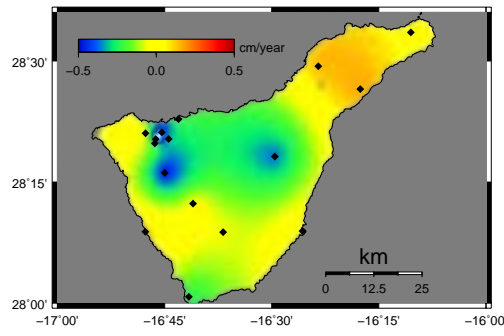

(b) east

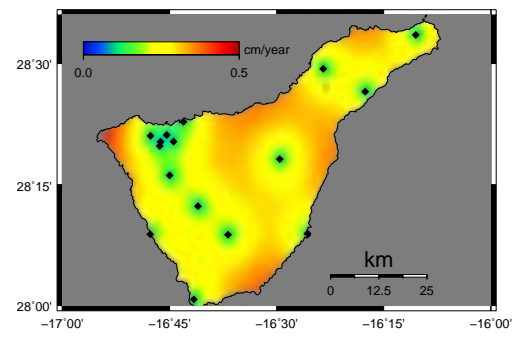

(e) east st. dev

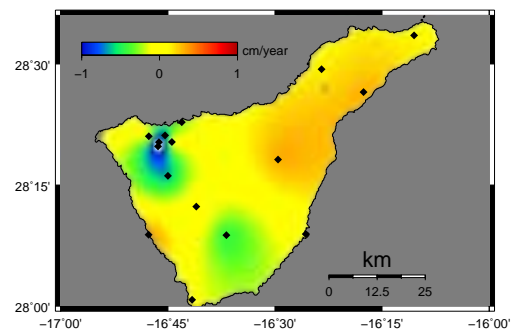

(c) up

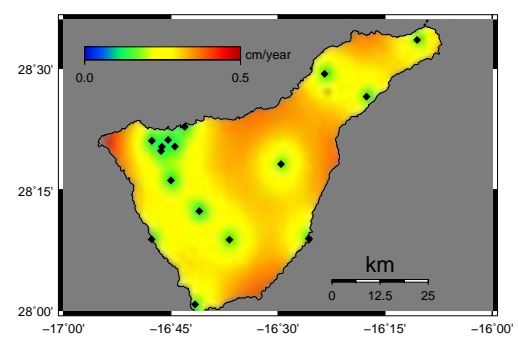

(f) up st. dev.

Fig. 4. Interpolated by ordinary kriging GPS velocities calculated from 2000-2006 campaign GPS time series applying linear regression. Black diamonds are location of GPS sites.

DInSAR data in order to increase signal to noise ratio and to reduce the contribution from atmospheric noise. Stacking was performed by setting a spatial reference point and multiple reference points were tested and no significant differences were observed. The RMS errors were also calculated for each stack (not presented here due to space limitation) which then were used as a weighting terms in the optimization. The synthetic interferograms calculated from interpolated GPS data were used to adjust static shifts of the DInSAR stacks.

Four optimization runs were performed for this region, which are summarized in Table II. First and third runs used only ascending stacks and the second and fourth runs used both, ascending and descending stacks. In this paper only displacements from the fourth run are presented on the Fig 5 due to space limitation. This run was chosen because it utilizes all GPS data from 2000 until 2006 and all ascending and descending interferograms, combined in two stacks. The GPS coverage of some of the campaigns concentrated around the NW part of the island, and therefore the accuracy of the final velocity fields is the highest in this area.

\section{RESULTS AND DISCUSSION}

Analysis of individual interferograms and interferometric stacks (Fig 3) suggests that some signal in the central part of the island correlates with the topography. It is anticipated that this signal could be caused by the water vapor noise from clouds and fog rather than errors in digital elevation model used for removing topography. However, the possibility of ground deformations caused by volcanic activities (or thermo elastic effects) can not be completely eliminated at this time.

The ordinary kriging technique used for interpolation of initial GPS velocities significantly depends on the type of the variogram model. In this work the variogram analysis of GPS velocities did not reveal any particular variogram trend and an exponential variogram model was chosen as an initial model for interpolation. Such behavior possibly can be explained by the complexity of ground deformations occurring on the island, and suggests that a denser GPS network is required.

The optimized velocity maps that were calculated using GPS data acquired between 2000 and 2006 and both, ascending and descending stacks, are presented on Figs 5(a), 5(b), 5(c) with corresponding errors 5(d), 5(e) and 5(f). In comparison to the original velocity maps derived from GPS data alone, the optimized velocity maps are not as smooth but the degree of changes affecting each component varies. This happens because InSAR is particularly sensitive to vertical motion (incidence angle is approximately 20 degrees) and, therefore, the InSAR contribution to the vertical velocity map is maximum during the optimization. The contribution to horizontal components depends on the azimuth of the satellite and in this case is moderate for eastern and minimal for northern component. Another factor affecting the degree of change is the initial accuracy of GPS data. Because the accuracy of GPS vertical component is lower than the accuracy of horizontal components it is weighted lower during the optimization.

The subsidence with a rate of a few $\mathrm{mm} /$ year is observed on both initial and optimized velocity maps in the NW corner of the island and some signal in the same region is observed on eastern component as well. It was discovered in previous work that at least some part of this subsidence is driven by extraction of groundwater, however, it is not observed on interferometric stacks used in this work. The possible explanation of this behavior is that it is related to a seasonal fluctuation of the groundwater levels and the long term subsidence trend reverses during rainy seasons when aquifers get recharged. This was confirmed by observing subsidence signal on some 


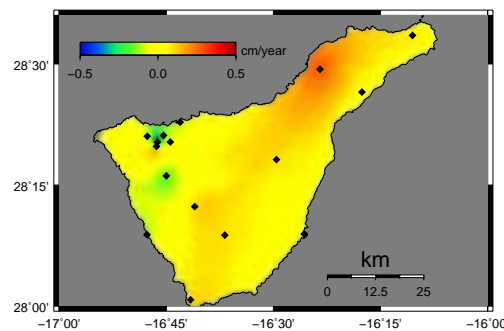

(a) north optimized

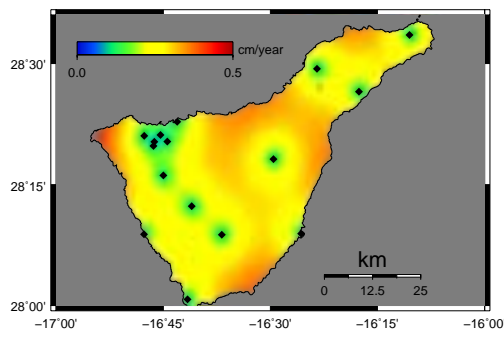

(d) north opt. st. dev.

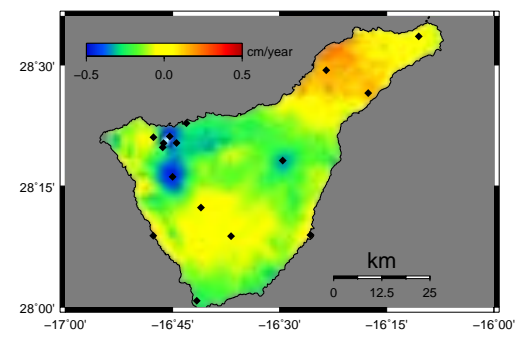

(b) east optimized

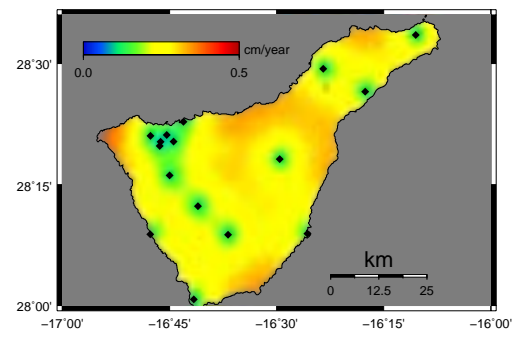

(e) east opt. st. dev.

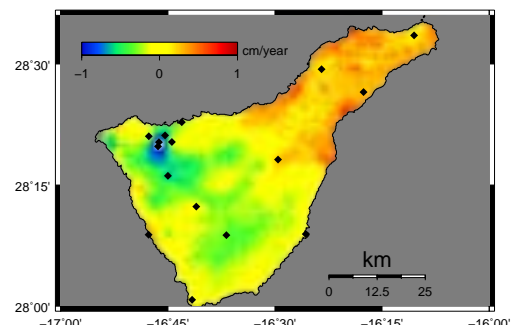

(c) up optimized

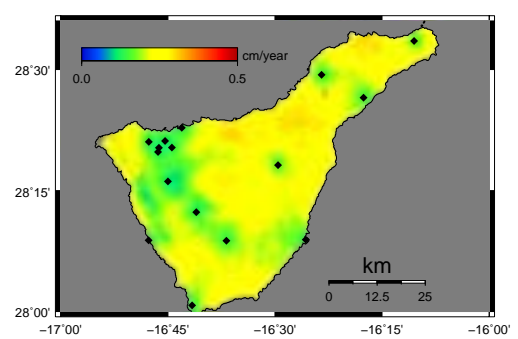

(f) up opt. st. dev.

Fig. 5. Velocity maps with corresponding errors calculated from 2000-2006 time series and ascending and descending interferometric stacks using GPSDInSAR optimization. Black diamonds are location of GPS sites.

interferograms that were used for stacking.

Additional signals were observed on optimized velocity maps and currently are being analyzed. These are not discussed here due to space limitations.

\section{CONCLUSION}

As discussed in this work, a new GPS-DInSAR optimization technique was used to combine 2000-2006 campaign GPS time series and ascending and descending interferometric stacks acquired by ENVISAT during approximately the same time. This technique illustrates a methodology that can combine various types of geodetic data into time-dependent densely spaced three-dimensional deformation field in order to identify local and regional geodetic signals. The technique can be expanded by adding other sorts of data such as LIDAR and leveling. Analysis of results revealed the complexity of GPS and DInSAR observations on Tenerife Island. For example, some subsidence signal in the NW corner of the island was observed only by GPS, while other signal in the center of the island was observed only by DInSAR.

\section{ACKNOWLEDGMENT}

This research was partially supported by the GEOMOD project (CGL2005-05500-C02). The ENVISAT precise orbits used in this work were provided by Delft University of Technology and the images were plotted with the help of GMT software developed and supported by Paul Wessel and Walter H. F. Smith. The work of KFT was accomplished under an NSERC Discovery Grant.

\section{REFERENCES}

[1] G. Ablay and P. Kerry. Gravity constraints on the structure and volcanic evolution of Tenerife, Canary Islands. Journal of Geophysical Research, $105: 5783-5796,2000$
[2] V. Arana, A. Camacho, F. Garcia, A. Montesinos, I. Blanco, R. Vieira, and A. Felpeto. Internal structure of Tenerife (Canary Islands) based on gravity aeromagnetic and volcanological data. J. Vol. Geotherm. Res. 103:43-64, 2000.

[3] J. Arnoso, J. Fernandez, R. Vieire, E. Velez, and A. Venedikov. Results of tidal observations in Tenerife, Canary Islands. Mar. Terr. Bull. Inf., 132:10283-10290, 2000.

[4] U. (edited by ) Hugentobler, S. Schaer, and P. Fridez. Bernese GPS software version 4.2. Astronomical institute, University of Berne, Switzerland, 2001.

[5] J. Fernandez, R. Romero, K. Carrasco, D. Tiampo, G. RodriguezVelasco, A. Aparicio, V. Arana, and F. Gonzalez-Matesanz. Detection of displacements on Tenerife Island, Canaries, using radar interferometry. Geophysical Journal International, 160:33-45, 2005.

[6] J. Fernandez, T. Yu, G. Rodrigez-Velasco, J. Gonzalez-Matesanz, R. Romero, G. Rodriguez, R. Quiros, A. Dalda, A. Aparico, and M. Blanco. Neo geodetic monitoring system in the volcanic island of Tenerife, Canaries, Spain. combination of InSAR and GPS techniques. J. Vol. Geotherm. Res., 124:241-253, 2003.

[7] A. Garcia, J. Vila, R. Ortiz, R. Marcia, R. Sleeman, J. Marrero, N. Sanchez, M. Tarraga, and A. Correig. Monitoring the reawakaning of the Canary Islands Teide volcano. Eos Trans. AGU, 87(6):61, 2006.

[8] J. Gottsman, L. Woller, J. Marti, J. Fernandez, A. Camacho, and P. Gonzalez. New evidence for reawakening of Teide volcano. Geophysical Research Letters, 33:L20311, 2006.

[9] P. Hernandez, N. Perez, J. Salazar, M. Sato, K. Notsu, and H. Wakita Soil gas $\mathrm{CO} 2, \mathrm{CH} 4$, and $\mathrm{H} 2$ distibution in and around Las Canadas Caldera, Tenerife, Canary Islands, Spain. J. Vol. Geotherm. Res. 103:425-438, 2000 .

[10] Almendros J., Ibanez J., Alguacil G., Morales J., Del Pezzo E., La Rocca M., Ortiz R., Arana V., and Blanco M.J. A double seismic antenna experiment at Teide volcano: existance of local seismicity and lack of evidences of volcanic tremor. J. Vol. Geotherm. Res., 103:439-462, 2000.

[11] N. Perez. Premonitory geochemical and geophysical signatures of volcanic unrest in tenerife, canary islands. In Geophysical Research Abstracts, 7, Abstract 09993, sref-id:EGU05-A-09993, 2005.

[12] J. Salazar, N. Perez, and Hernandez. P. Secular variations of soil $\mathrm{CO} 2$ flux levels at the summit cone of Teide volcano, Tenerife, Canary Islands. EOS Trans., AGU, 81:48, 2000.

[13] S. Samsonov and K. Tiampo. Analytical optimization of DInSAR and GPS dataset for derivation of three-dimensional surface motion Geoscience and remote sensing letters, 3(1):107-111, 2006. 
[14] S. Samsonov, K. Tiampo, and J. Rundle. Application of DInSARGPS optimization for derivation of three dimensional surface motion of southern California region along the San Andreas fault. Computers and Geoscienses, 34(5):503-514, 2008.

[15] S. Samsonov, K. Tiampo, J. Rundle, and Z. Li. Application of DInSARGPS optimization for derivation of fine scale surface motion maps of southern California. Transactions on geoscience and remote sensing, 45(2):512-521, 2007.

[16] M. Sevilla and F. Sanchez. Geodetic network for deformation monitoring in the Caldera of Teide. The European laboratory volcanoes. In $\mathrm{R}$. Casale, editor, In proceedings of the second workshop European Commission, pages 615-636, 1996.

[17] R. Vieira, C. Toro, and V. Arana. Microgravimetric survey in the Caldera of Teide, Tenerife, Canary Islands. Tectonophysics, 130:249-257, 1986.

[18] T. Yu, J. Fernandez, C. Tseng, M. Sevilla, and V. Arana. Sensitivity test of the geodetic network in Las Canadas Caldera, Tenerife for volcano monitoring. J. Vol. Geotherm. Res., 103:393-407, 2000. 